\title{
Designing Nanostructures for Phonon Transport via Bayesian Optimization
}

\author{
Shenghong Ju, ${ }^{1,2}$ Takuma Shiga, ${ }^{1,2}$ Lei Feng, ${ }^{1}$ Zhufeng Hou, ${ }^{2}$ Koji Tsuda, ${ }^{2,3,4}$ and Junichiro Shiomi ${ }^{1,2,5, *}$ \\ ${ }^{1}$ Department of Mechanical Engineering, The University of Tokyo, \\ 7-3-1 Hongo, Bunkyo, Tokyo 113-8656, Japan \\ ${ }^{2}$ Center for Materials research by Information Integration, National Institute for Materials Science, \\ 1-2-1 Sengen, Tsukuba, Ibaraki 305-0047, Japan \\ ${ }^{3}$ Department of Computational Biology and Medical Sciences, Graduate School of Frontier Sciences, \\ The University of Tokyo, 5-1-5, Kashiwanoha, Kashiwa, Chiba 277-8561, Japan \\ ${ }^{4}$ RIKEN Center for Advanced Intelligence Project, 1-4-1 Nihombashi Chuo-ku, 103-0027 Tokyo, Japan \\ ${ }^{5}$ Core Research for Evolutional Science and Technology (CREST), \\ Japan Science and Technology Agency, 4-1-8, Kawaguchi, Saitama 332-0012, Japan
}

(Received 2 September 2016; revised manuscript received 13 January 2017; published 17 May 2017)

\begin{abstract}
We demonstrate optimization of thermal conductance across nanostructures by developing a method combining atomistic Green's function and Bayesian optimization. With an aim to minimize and maximize the interfacial thermal conductance (ITC) across $\mathrm{Si}-\mathrm{Si}$ and $\mathrm{Si}-\mathrm{Ge}$ interfaces by means of the $\mathrm{Si} / \mathrm{Ge}$ composite interfacial structure, the method identifies the optimal structures from calculations of only a few percent of the entire candidates (over 60000 structures). The obtained optimal interfacial structures are nonintuitive and impacting: the minimum ITC structure is an aperiodic superlattice that realizes $50 \%$ reduction from the best periodic superlattice. The physical mechanism of the minimum ITC can be understood in terms of the crossover of the two effects on phonon transport: as the layer thickness in the superlattice increases, the impact of Fabry-Pérot interference increases, and the rate of reflection at the layer interfaces decreases. An aperiodic superlattice with spatial variation in the layer thickness has a degree of freedom to realize optimal balance between the above two competing mechanisms. Furthermore, the spatial variation enables weakening the impact of constructive phonon interference relative to that of destructive interference. The present work shows the effectiveness and advantage of material informatics in designing nanostructures to control heat conduction, which can be extended to other nanostructures and properties.
\end{abstract}

DOI: 10.1103/PhysRevX.7.021024

Subject Areas: Condensed Matter Physics

\section{INTRODUCTION}

The exploration and design of materials with desired thermal transport properties hold importance in thermal management applications such as thermal interface materials [1], heat pipe [2,3], thermoelectrics [4], and thermal insulator [5]. As the length scales of materials are reduced to nanoscale, phonon transport becomes more ballistic (or quasiballistic) and the interfacial thermal conductance (ITC) determines the heat conduction through the entire material $[6,7]$. In other words, the heat conduction becomes controllable through manipulating the interface structure. Various individual factors for tuning the ITC have been reported, such as roughness [8,9], vacancy defects [10], lattice orientation [11,12], nanoinclusions [13], and interfacial adhesion or bonding [14,15]. However, these factors

\footnotetext{
* Corresponding author. shiomi@photon.t.u-tokyo.ac.jp

Published by the American Physical Society under the terms of the Creative Commons Attribution 4.0 International license. Further distribution of this work must maintain attribution to the author(s) and the published article's title, journal citation, and DOI.
}

are usually coupled with each other and are sensitive to the detailed atomic configurations, which makes it hard to identify the total controllability of ITC. The search for optimal structure becomes even more difficult in the case of structures with multiple interfaces, such as superlattices [16-19], nanocrystals [20], and nanocomposites [21,22], where the constructive and destructive phonon interference and resonance effects make the heat transport more complicated. The key next-generation technology here may be materials informatics [23-25]: integration of material property calculations or measurements and informatics to accelerate the material discovery and design.

During the past decade, informatics has been successfully applied in designing drugs [26,27], polymers [28], and grain boundaries [29], and also in guiding experiments [30]. In the field of heat transfer, materials informatics has been applied to search for thermoelectric materials with low thermal conductivity from developed material databases [31,32]. Optimal distribution of nanoparticle size for minimum thermal conductivity was also performed by using a kind of evolutionary algorithm [33]. However, the nanostructure optimization for thermal transport is still in its infancy. Developing an effective optimization method for designing nanostructures is necessary and has great 
potential for application. In this work, we develop a framework by means of atomistic Green's function (AGF) [8,34-36] and Bayesian optimization methods [37] and demonstrate the efficiency and ability to identify nonintuitive interfacial structures that realize maximum and minimum ITC.

\section{METHODOLOGY}

We explain the basis of the method by taking a problem to design the composite interfacial structure to tune heat conduction across the $\mathrm{Si}-\mathrm{Si}$ and $\mathrm{Si}-\mathrm{Ge}$ interfaces. As illustrated in Fig. 1, the system consists of an interfacial region between two materials $A(\mathrm{Si})$ and $B(\mathrm{Si}$ or $\mathrm{Ge})$ with infinite thickness. As for the transverse direction, a supercell approach is adopted to simulate an interface with infinite cross section. The lattice mismatch between $\mathrm{Si}$ and Ge is ignored since the stress effect due to the lattice mismatch has negligible effect on thermal transport [35]. The interfacial structure consists of either $\mathrm{Si}$ or $\mathrm{Ge}$, and the optimization problem is how to arrange the $\mathrm{Si}$ and $\mathrm{Ge}$ atoms to obtain the largest and smallest ITC.

Four basic elements are required when conducting material informatics: the descriptor, evaluator, calculator, and optimization method. The descriptors are used to describe the possible structure candidates considered during the optimization. In this study, we use a binary flag to describe the state of each atom: "1" and " 0 " represent the $\mathrm{Ge}$ and $\mathrm{Si}$ atom, respectively. As for the evaluator, the value of ITC is chosen to quantitatively evaluate the performance of each configuration.

The AGF method [8,34-36] was employed to calculate the phonon transmission function $\Xi(\omega)$,

$$
\Xi(\omega)=\operatorname{Tr}\left[\Gamma_{L} G^{r} \Gamma_{R} G^{a}\right],
$$

where $\omega$ is the phonon frequency, $G^{r}$ and $G^{a}$ are the retarded and advanced Green functions of the scattering region, the level broadening matrices $\Gamma_{L}=i\left(\Sigma_{L}^{r}-\Sigma_{L}^{a}\right)$ and $\Gamma_{R}=i\left(\Sigma_{R}^{r}-\Sigma_{R}^{a}\right)$ describe the rates of inflow from the left lead and outflow into the right lead, and $\Sigma_{L}$ and $\Sigma_{R}$ are the self-energies, which are calculated from surface Green's functions of left and right leads, respectively. The surface Green's function is calculated for semi-infinite regions, reproducing materials $A$ and $B$ with infinite thickness in Fig. 1, and thus, the transmission can be calculated for the full phonon spectrum including long wave phonons.

With the two leads kept at different temperatures $T_{L}$ and $T_{R}$, the heat current flowing through the device is given by the Landauer formula [38],

$$
J=\frac{1}{2 \pi} \int \hbar \omega\left[f_{L}\left(\omega, T_{L}\right)-f_{R}\left(\omega, T_{R}\right)\right] \Xi(\omega) d \omega,
$$

where $f_{L}$ and $f_{R}$ are the Bose-Einstein distributions of phonons. In the limit of small temperature differences, the value of ITC $(\sigma)$ at average temperature $T$ can be further obtained by

$$
\sigma=\frac{\hbar^{2}}{2 \pi k_{B} T^{2} S} \int \omega^{2} \Xi(\omega) \frac{e^{\hbar \omega / k_{B} T}}{\left(e^{\hbar \omega / k_{B} T}-1\right)^{2}} d \omega,
$$

where $k_{B}$ is the Boltzmann constant and $S$ is the cross-sectional area. In this work, the AGF calculation is conducted by using the Atomistix ToolKit simulation package (ATK) [39] with Tersoff potentials [40,41]. The size of the supercell in the transverse direction (perpendicular to the direction of heat conduction) is 1 unit cell (UC); i.e., $5.43 \AA \times 5.43 \AA$. The number of transverse $k$ points is selected as $20 \times 20$, which has been tested (See Fig. S1 in Supplemental Material [42]) to ensure convergence of the transmission calculation.

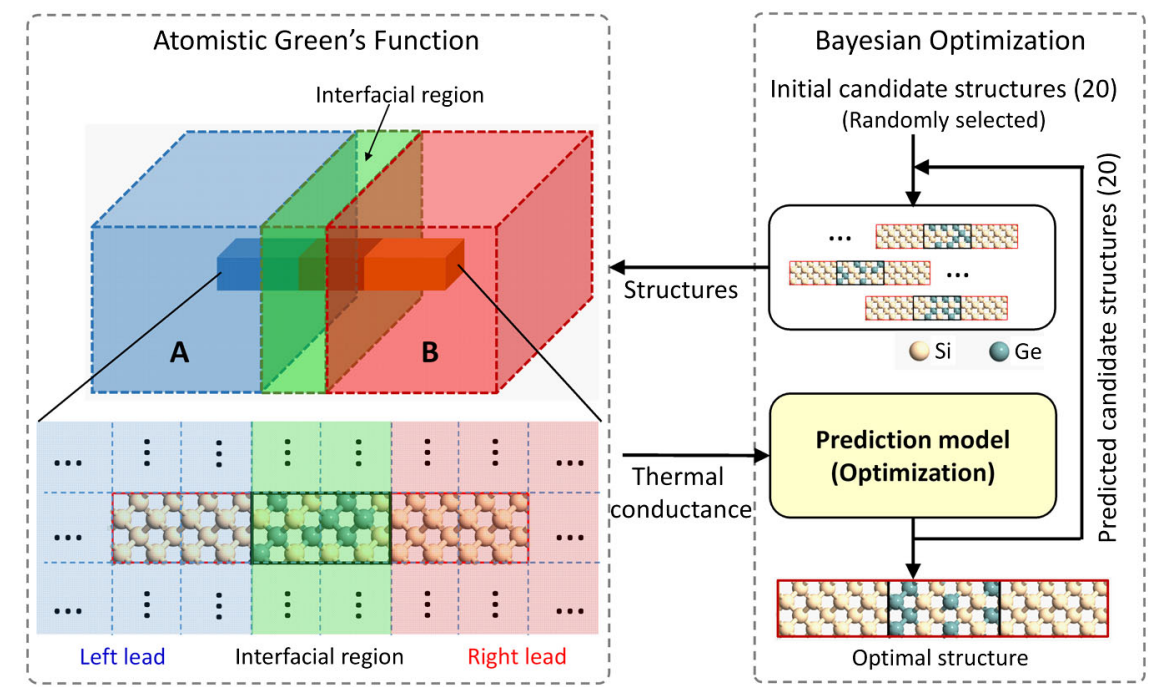

FIG. 1. Schematics of the materials informatics method combing the atomistic Green function (AGF) and Bayesian optimization. In the actual AGF calculation, the buffer region is installed between the interfacial region and the lead, which is omitted in the sketch for simplicity. 
We employ our developed open-source Bayesian optimization library сомво [37] to perform the optimization process. Bayesian optimization is an experimental design algorithm based on machine learning [43]. As shown in Fig. 1, suppose that ITCs of $n$ candidates are initially calculated, and we are to choose the next one to calculate. A Bayesian regression function is learned from $n$ pairs of descriptors and ITCs (i.e., training examples). For each of the remaining candidates, a predictive distribution of ITCs is estimated. The best candidate is chosen based on the criterion of expected improvement [43]. Finally, ITC is calculated for the chosen candidate, and it is added to the training examples. By repeating this procedure, the calculation of ITCs is scheduled optimally, and the best candidate can be found quickly.

As the prediction model, we employ a Bayesian linear regression model combined with a random feature map,

$$
y=\boldsymbol{w}^{T} \varphi(\boldsymbol{x})+\varepsilon,
$$

where $\boldsymbol{x}$ is a $d$-dimensional vector corresponding to a candidate, $\boldsymbol{w}$ is a $D$-dimensional weight vector, and $\varepsilon$ is the noise subject to normal distribution with mean 0 and variance $\sigma$. The random feature map is chosen so that the inner product corresponds to the Gaussian kernel [44]:

$$
\varphi(\boldsymbol{x})^{T} \varphi\left(\boldsymbol{x}^{\prime}\right)=\exp \left(-\frac{\left\|\boldsymbol{x}-\boldsymbol{x}^{\prime}\right\|^{2}}{\eta^{2}}\right) .
$$

The performance of the model depends on hyperparameters $\sigma$ and $\eta$. In Сомво, they are initialized according to a heuristic procedure by Yang et al. [45]. At every round with 20 training examples added, the hyperparameters are periodically configured and updated by maximizing the type-II likelihood [46].

\section{RESULS AND DISCUSSIONS}

We formulate two optimization problems: the first part is optimization of a relatively small interfacial region accounting for full degrees of freedom to demonstrate the validity and capability of the current method, and the second part is optimization of a larger interfacial region with layered structures. In the first part, the thickness of the interfacial region is fixed at $10.86 \AA$ ( 8 atomic layers). This makes the total number of possible candidates $C_{16}^{8}=12870$. Note that the computational load of this part is made small enough so that we can calculate all of the candidates to validate the optimal structure and the efficiency. Figures 2(a)-(d) show the resulting optimal structures with minimum and maximum ITC for $\mathrm{Si}-\mathrm{Si}$ and $\mathrm{Si}-\mathrm{Ge}$ interfaces.

To test the performance of Bayesian optimization, 10 rounds of optimization are conducted with different initial choices of 20 candidates. As shown in Figs. 2(e) and 2(f), all optimizations come to convergence within calculations of 438 structures, which is only $3.4 \%$ of the total number of candidates (12870). To check the accuracy of the optimization, the ITCs of all candidates are also calculated, and the maximum and minimum ITC and the corresponding structures are confirmed to be exactly the same as those obtained by Bayesian optimization. The probability distributions of the ITCs obtained by calculation of all the candidates shown in the insets of Figs. 2(e) and 2(f) confirm that the probabilities decrease as ITC values approach the minimum and maximum, indicating that the current problem is suited for optimization. The ratios of maximum to minimum ITC for Si-Si and Si-Ge interfaces are 2.4 and 2.2 , respectively, which indicates that the ITC significantly depends on the interfacial nanostructure.

Figures 2(g) and 2(h) compare the phonon transmission function of optimal structures with those of bare Si-Si and $\mathrm{Si}-\mathrm{Ge}$ interfaces. Note that the bare $\mathrm{Si}-\mathrm{Si}$ interface here means the absence of interface. For phonon frequency lower than $3 \mathrm{THz}$, the transmissions of different structures are almost the same because the transport of long wavelength phonons is not sensitive to the interfacial structures with smaller length scales. On the other hand, for higher phonon frequency, the transmission function strongly depends on the structure. The optimal structure with maximum ITC for the Si-Si interface shown in Fig. 2(a) is intuitive as the structure provides a continuum path of $\mathrm{Si}$ for phonons to coherently propagate. However, the other three optimal structures shown in Figs. 2(b)-2(d) are not intuitive. The optimal structure with maximum ITC for Si-Ge interfaces shown in Fig. 2(b) can be considered as a kind of rough interface, and the phonon transmission is clearly enhanced at frequency ranges 4-5 and 8-11 THz. This agrees with the previous AGF calculation result on a rough interface [8], which showed that the roughness can enhance the phonon transmission at the interface. Note that similar enhancement of conductance by larger scale roughness has been reported using molecular dynamics simulations [9].

The structures with minimum ITC for both the Si-Si and Si-Ge interface shown in Figs. 2(c) and 2(d) are aperiodic superlattices with the constituent layers perpendicular to the direction of heat conduction. The structure is different from periodic superlattices, which have been widely studied as a class of materials with low thermal conductivity motivated particularly by thermoelectric applications. Their thermal conductivity has been found to take a minimum with respect to the layer thickness due to the crossover between wave interference and particle scattering of phonons at the periodic interlayer interfaces [17]. As shown in a recent study, aperiodic superlattices can have lower thermal conductivity than the periodic ones [47]. However, as the thickness of each layer in the superlattice is different, the underlying physics becomes complicated and identifying the optimal structure from the vast number of candidates based on physical principles has been a challenge. 
(a) Si-Si, max ITC

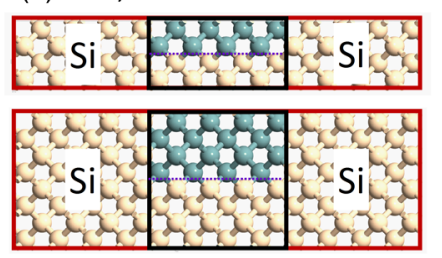

(c) Si-Si, min ITC

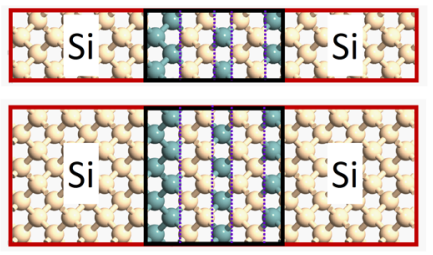

(e)

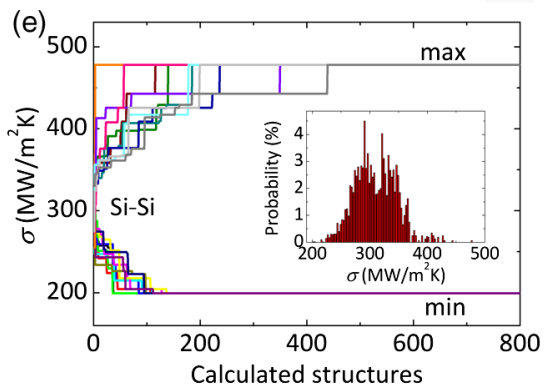

(g)

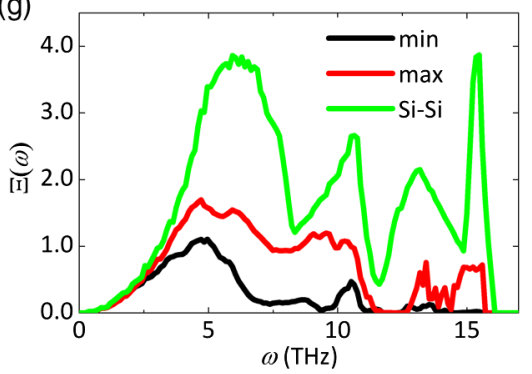

(b) Si-Ge, max ITC

$1 \cup C \times 1 \cup C$

$2 \mathrm{UC} \times 2 \mathrm{UC}$

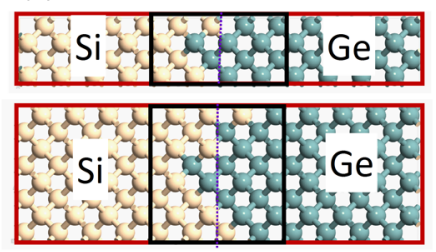

(d) Si-Ge, min ITC

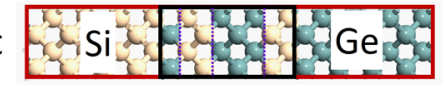

$2 U C \times 2 \cup C$

Si

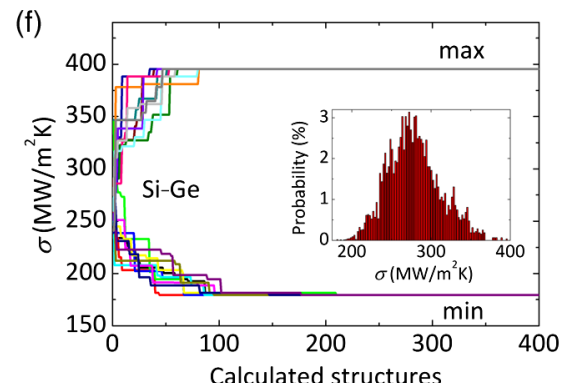

(h)

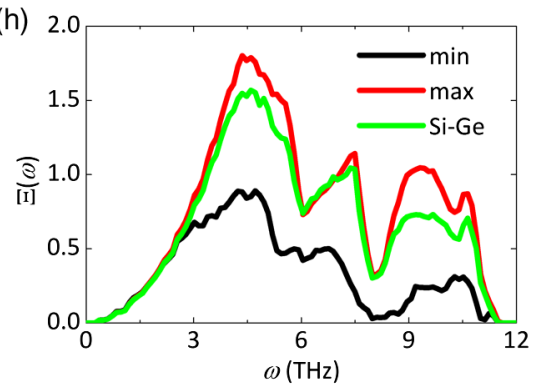

FIG. 2. Interfacial Si/Ge alloy structure optimization. (a)-(d) Optimal structures with the maximum and minimum interfacial thermal conductance (ITC) for Si-Si and Si-Ge interface with different sizes of supercell cross section $(1 \mathrm{UC} \times 1 \mathrm{UC}$ and $2 \mathrm{UC} \times 2 \mathrm{UC})$. The thickness of the interfacial region is $1.1 \mathrm{~nm}$. (e),(f) The 10 optimization runs with different initial choices of candidates, where the insets show the probability distributions of ITC obtained from calculations of all the candidates. $(\mathrm{g}),(\mathrm{h})$ Phonon transmission functions of the optimized interfacial structure (maximum and minimum ITC) and bare Si-Si (i.e., no interfacial structure) and Si-Ge interfaces.

Since the current transverse supercell approach accounts only for structures with the supercell periodically repeated in the transverse direction (Fig. 1), the generality of the above optimal structures found with the $1 \mathrm{UC}$ transverse supercell can be questioned. To this end, we perform the optimization with a larger cross section, $2 \mathrm{UC} \times 2 \mathrm{UC}$. Here, since blindly performing the optimization with all degrees of freedom would explode the number of candidates to $\sim 10^{18}$, we reduce the number of candidates based on the knowledge learned from the smaller cross-section case (details are described in Supplemental Material [42]). As shown in Figs. 2(c) and 2(d), the optimal structures for minimum conductance of $\mathrm{Si}-\mathrm{Si}$ and $\mathrm{Si}-\mathrm{Ge}$ interfaces are exactly the same and are independent of the supercell size.
This is understandable since the optimal structures in these cases are layered structures with no variation in the transverse directions. On the other hand, the optimal structures for maximum conductance depend on the supercell size. In the case of Si-Ge interface, the smaller supercell calculation captures only a part of the optimal structure obtained by the larger supercell calculation. In the case of $\mathrm{Si}-\mathrm{Si}$ interface, however, the general feature that half of the supercell is filled with $\mathrm{Si}$ to provide a continuum path for phonon transport remains the same for both supercell sizes.

Based on the knowledge learned above, i.e., layered structures give rise to minimum conductance and do not depend on the size of transverse supercell, we move on to the second system and extend the ITC minimization 


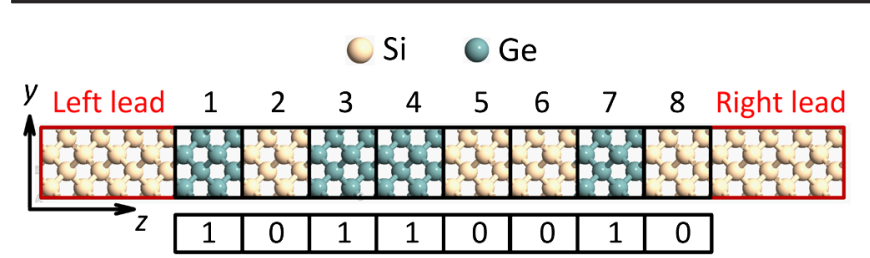

Si/Ge superlattice region

FIG. 3. Sketch of the 8-UL superlattice structure with equal numbers of $\mathrm{Si}$ and $\mathrm{Ge}$ atoms at $\mathrm{Si}-\mathrm{Si}$ interface to illustrate the descriptor. Eight binary flags are used to indicate the specie of each UL ("1" for Ge and "0" for Si), and thus, the descriptor of this structure is (10110010).

problem of interfacial structure to a realistic setup by enlarging the length scales of the structure to the extent controllable in the experiments [48]: the thickness of the unit layer (UL) is $5.43 \AA$, and total thickness of the interfacial structure ranges from 8 to $16 \mathrm{UL}$ (from 4.35 to $8.69 \mathrm{~nm}$ ). To compensate for the increase in the number of candidates, we limit the candidates to $\mathrm{Si} / \mathrm{Ge}$ superlattices (periodic and aperiodic) based on the above finding that the alloy structure with minimum ITC is a superlattice. Figure 3 illustrates the 8-UL superlattice structure in the case of Si-Si interface. Similarly to the descriptors used in the alloy structure optimization, 8 binary flags are used to indicate the state of each UL (" 1 " indicates Ge and " 0 " indicates $\mathrm{Si}$ ). For instance, the descriptor for the case shown in Fig. 3 is (10110010).

Table I lists all of the optimal superlattice structures for $\mathrm{Si}-\mathrm{Si}$ and $\mathrm{Si}-\mathrm{Ge}$ interfaces optimized for various total thickness. For each layer thickness, the optimization is performed for an equal and variable fraction of $\mathrm{Si} / \mathrm{Ge}$ atoms. The number of candidates in each case is also listed in brackets. As expected, the numbers of candidates in case of variable fraction are much larger than those in the case of a fixed (and equal) fraction. It can be seen that the optimal superlattice always begins or ends with a different material layer from the lead region, which is understandable because it enhances the phonon scattering. Figure 4(a) summarizes the minimum ITC values versus the number of ULs (i.e., total thickness of the interfacial structure). As the number of UL increases, the minimum ITC decreases. For Si-Si interfaces, the minimum ITC of the variable-fraction case is smaller than that of the fixed-fraction case, while for Si-Ge interfaces, the difference between the two cases is very small. Figure 4(b) compares the ITC of the obtained aperiodic superlattice at the $\mathrm{Si}-\mathrm{Si}$ interface with that of traditional periodic superlattices (10) with the best period thickness (optimized separately for each total length). We find that the ITC of the designed aperiodic superlattices with a fixed $\mathrm{Si} / \mathrm{Ge}$ fraction is significantly smaller (by $20 \%-50 \%$ ). Not to mention, making the fraction variable can further reduce the ITC.

Now that the optimal structures are identified, we look into the mechanisms behind the small ITC. The first obvious attempt is to see it from the view of phonon dispersion relations and phonon density of states (DOS), as is broadly done to discuss phonon interference. Taking the 10-UL superlattice structures as an example, we choose three typical structures for comparison: the obtained optimal structure (1101010001), the periodic superlattices with minimum layer thickness (1010101010), and maximum layer thickness (1111100000). Figures 4(c) and 4(d) show the phonon dispersion, phonon DOS, and phonon transmission function. Comparing the phonon dispersion relations of the structures (1101010001) and (111110000) [Fig. 4(c)], the difference is quite small even though their ITC values differ by a factor of 2 . The DOS of the three structures are also almost the same except for some minor differences in the local peaks. However, we can see obvious differences in the phonon transmission function shown in Fig. 4(d), and the optimal structure clearly exhibits the minimum transmission. These suggest the incapability of phonon dispersion or DOS in explaining the mechanism of minimum ITC in the optimal structure.

We therefore take another route by breaking the characteristics of the structure into the thickness of a single layer (layer thickness) and the number of interfaces in the superlattice. For this, we construct model systems that allow us to independently vary the layer thickness and the number of interfaces, as shown in Figs. 5(a) and 5(c). In the

TABLE 1. Optimal superlattice structures obtained through Bayesian optimization for different setups and constraints. The numbers in the brackets are the number of candidates in each case. Thickness of a unit layer (UL) is $5.43 \AA$. The descriptors " 1 " and " 0 " describe Si and Ge UL.

\begin{tabular}{lcccc}
\hline \hline & $\mathrm{Si}-\mathrm{Si}$ & $\mathrm{Si}-\mathrm{Si}$ & $\mathrm{Si}-\mathrm{Ge}$ & $\mathrm{Si}-\mathrm{Ge}$ \\
\hline $\begin{array}{l}\text { Number } \\
\text { of ULs }\end{array}$ & fixed Si/Ge fraction & $\begin{array}{c}\text { variable Si/Ge } \\
\text { fraction }\end{array}$ & $\begin{array}{c}\text { fixed Si/Ge } \\
\text { fraction }\end{array}$ & $\begin{array}{c}\text { variable } \mathrm{Si} / \mathrm{Ge} \\
\text { fraction }\end{array}$ \\
\hline 8 & $11000101(70)$ & $11101101(256)$ & $10100110(70)$ & $10100110(256)$ \\
10 & $1101010001(252)$ & $1110110101(1024)$ & $100010110(252)$ & $100010110(1024)$ \\
12 & $101100100101(924)$ & $110110101001(4096)$ & $100101010110(924)$ & $100101010110(4096)$ \\
14 & $11011000101001(3432)$ & $11001010110111(16384)$ & $10011001010110(3432)$ & $10010101101110(16384)$ \\
16 & $1100010010110101(12870)$ & $1100101110110101(65536)$ & $1010110110010010(12870)$ & $1001010101101110(65536)$ \\
\hline \hline
\end{tabular}



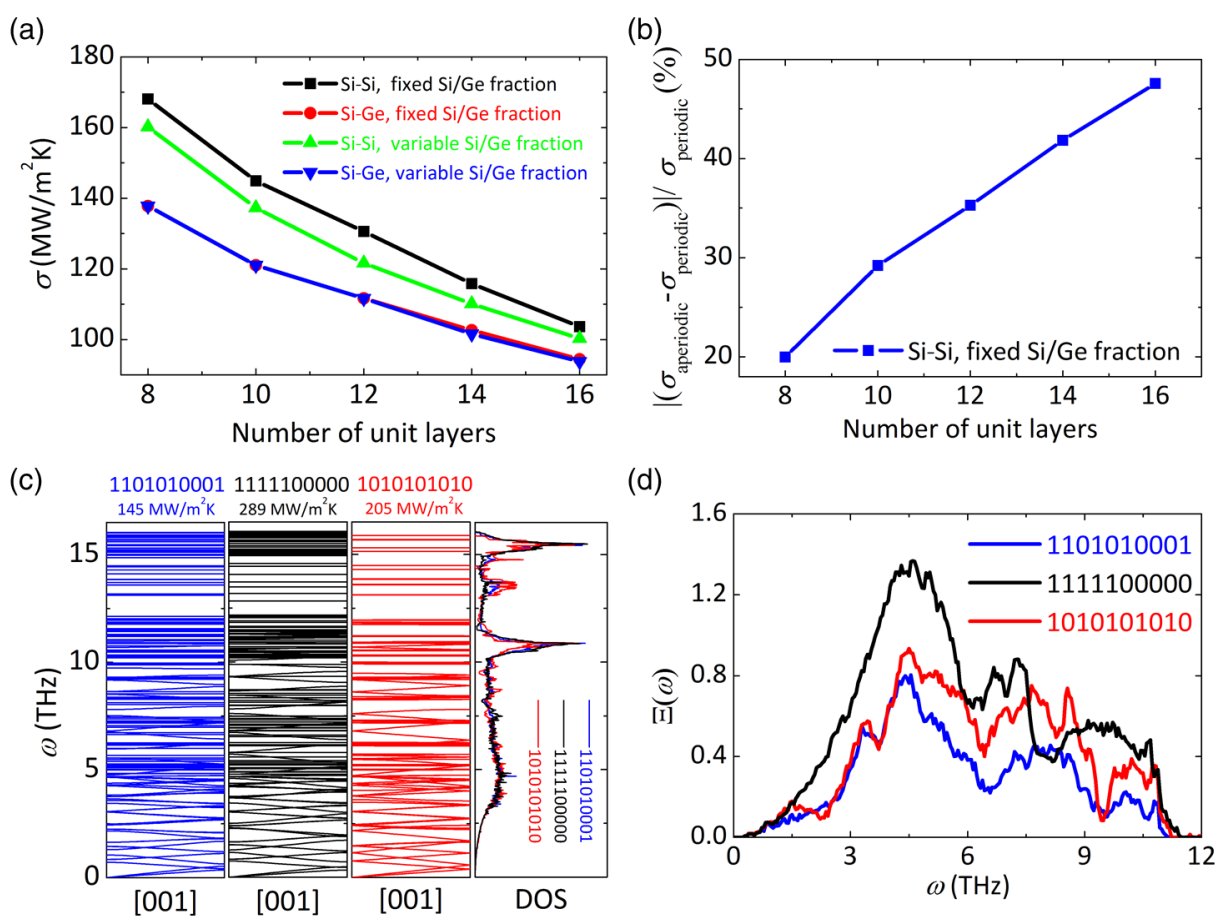

FIG. 4. (a) Comparison of ITC of interfacial structures at $\mathrm{Si}-\mathrm{Si}$ and $\mathrm{Si}-\mathrm{Ge}$ interfaces with variable ULs. (b) The reduction ratio $\left|\left(\sigma_{\text {aperiodic }}-\sigma_{\text {periodic }}\right)\right| / \sigma_{\text {periodic }}$, where $\sigma_{\text {aperiodic }}$ is the ITC of the optimized aperiodic superlattice, and $\sigma_{\text {periodic }}$ is the ITC of the corresponding periodic superlattice with period thickness optimized for each number of ULs (i.e., total length). The comparison of (c) the phonon dispersion and DOS, and (d) the phonon transmission for three superlattice structures optimal aperiodic superlattice (1101010001), and periodic superlattice with the largest (1111100000) and smallest (1010101010) periodic thickness.

former, the layer thickness is varied by fixing the number of interfaces, and in the latter, the number of interfaces is varied by fixing the length per layer. Figures 5(a) and 5(c) show that, as the layer thickness and number of thickness increase, the ITC decreases and eventually asymptotically converges to a constant value, which is consistent with the trends seen in former investigations of $\mathrm{Si} / \mathrm{Ge}$ structures $[35,49]$. The corresponding phonon transmission functions in Figs. 5(b) and 5(d) show that the general dependence on the layer thickness and number of thickness follows that of ITC.

The dependence on number of interface in Fig. 5(c) is intuitive since the scattering increases with the number of interface. The reason for the convergence will be discussed later. To understand the dependence on the layer thickness in Fig. 5(a), we adopt the 1D atomic chain model and calculate phonon transmission by changing the thickness of the scattering region $L$ (see Fig. S2 in Supplemental Material [42]). The results clearly exhibit the FabryPérot oscillations [50,51], with both constructive and destructive phonon interferences, which give rise to the strong dependence of phonon transmission function on the distance between two interfaces. When $L$ is small, the resonance occurs at specific frequencies; as $L$ increases, the number of resonant frequencies increases and eventually covers the entire frequency range. As a result, thermal conductance decreases faster when $L$ is small and eventually saturates to a constant value as $L$ increases. This trend resembles that of the Si/Ge system in Fig. 4(a), and thus, we attribute the observed trend to the Fabry-Pérot oscillations. This is in line with the discussion in Refs. $[35,49]$ in terms of coupling and decoupling of phonon waves.

On considering a superlattice with a given total thickness, the layer thickness and number of interfaces are two competing parameters, and this gives rise to the optimal structure with minimum ITC. On optimizing the balance between layer thickness and number of interfaces, an aperiodic superlattice can be superior to its periodic counterpart because it has a degree of freedom to spatially distribute parts with different layer thicknesses and numbers of interfaces.

To further highlight the above-discussed competition of the two effects, the ITCs of all the candidates are calculated for the 14-UL superlattice with fixed (equal) $\mathrm{Si} / \mathrm{Ge}$ fraction and the 10-UL superlattice with variable fraction. The ITC versus the number of interfaces in the superlattices is shown in Figs. 5(e) and 5(f). In both cases, the profile of ITCs with respect to the number of interfaces takes a minimum, which confirms the competition. Figures 5(e) and 5(f) also show that structures with the same number of interfaces can result in significantly different ITC due to the difference in the thickness of the constituent layers. Since the impact of the phonon scattering should remain the same for a fixed 
(a)

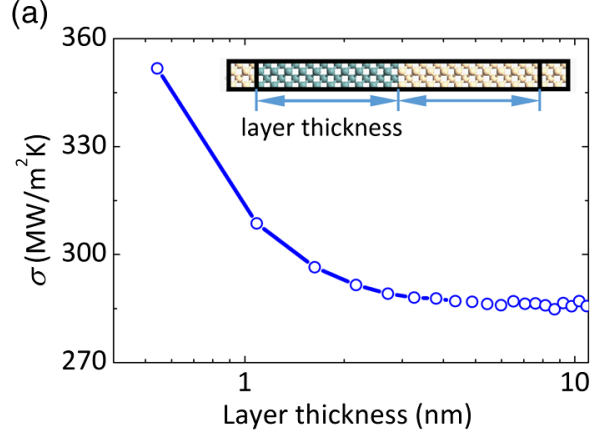

(c)

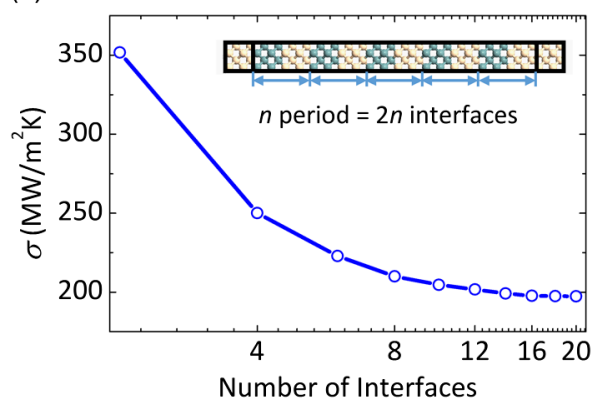

(e)

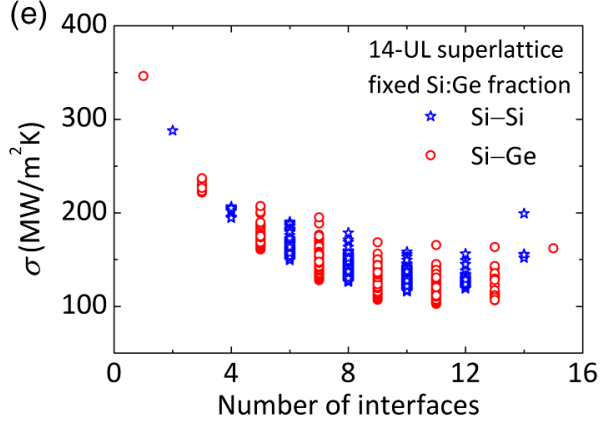

(b)

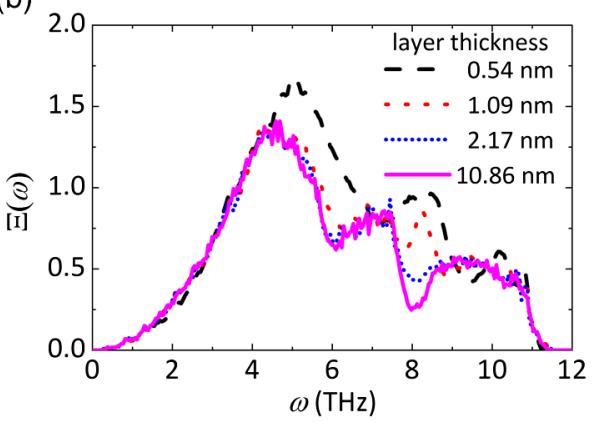

(d)

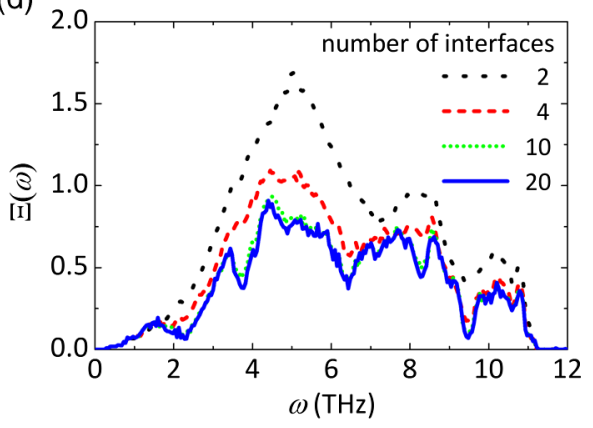

(f)

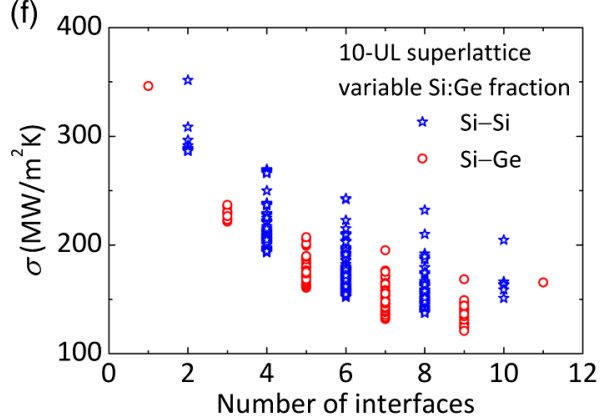

FIG. 5. (a),(b) ITC and phonon transmission versus the layer thickness. (c),(d) ITC and phonon transmission versus the number of interfaces with layer thickness of $1 \mathrm{UL}$, where $1 \mathrm{UL}$ is $5.43 \AA$ thick. (e),(f) ITC versus number of interfaces for cases of 14-UL superlattice with equal $\mathrm{Si} / \mathrm{Ge}$ layer number and 10 -UL superlattice with variable $\mathrm{Si} / \mathrm{Ge}$ fraction.

number of interfaces, the fact that the spread of the ITC is comparable to the magnitude of the ITC indicates that impact of the Fabry-Pérot resonance is as large as that of phonon scattering.

Now, to gain a deeper understanding of the physics of phonon transport in the optimized structure, we look into the role of phonon coherence, which can cause constructive and destructive interferences. To this end, we attempt to separate the transmission due to the particle (incoherent) effect and the wave (coherent) effect in superlattices by comparing the phonon transmission from the full AGF calculation and the cascade transmission model in view of the particle transport [52-54]. Note that phonon reflection at an interface itself gives rise to thermal resistance, but further interference of phonons (involving phonons reflected at different interfaces) gives rise to additional thermal resistance. Here, we describe the former as the particle (incoherent) effect because it does not matter whether the phonon is coherent or not, and the latter as the wave (coherent) effect because interference requires phonons to be coherent (i.e., can only be described by the wave picture). In the cascade model, the phonon transmission of each interface is assumed to be independent from each other, and the effective phonon transmission $\Xi_{\text {cascade }}$ is obtained as

$$
\frac{1}{\Xi_{\text {cascade }}}=\sum_{i} \frac{1}{\Xi_{i}}-\frac{N-1}{\Xi_{\mathrm{Si}}}
$$

where $N$ is the number of interfaces, $\Xi_{i}$ is the transmission coefficients of the $i$ th interface, and $\Xi_{\mathrm{Si}}$ is the phonon transmission of a perfect silicon crystal. Figure 6(a) compares the results obtained by the cascade model and full AGF calculation for periodic superlattice with three different numbers of periods $(2,4$, and 6$)$, where a period consists of one Si UL and one Ge UL ("10" in 

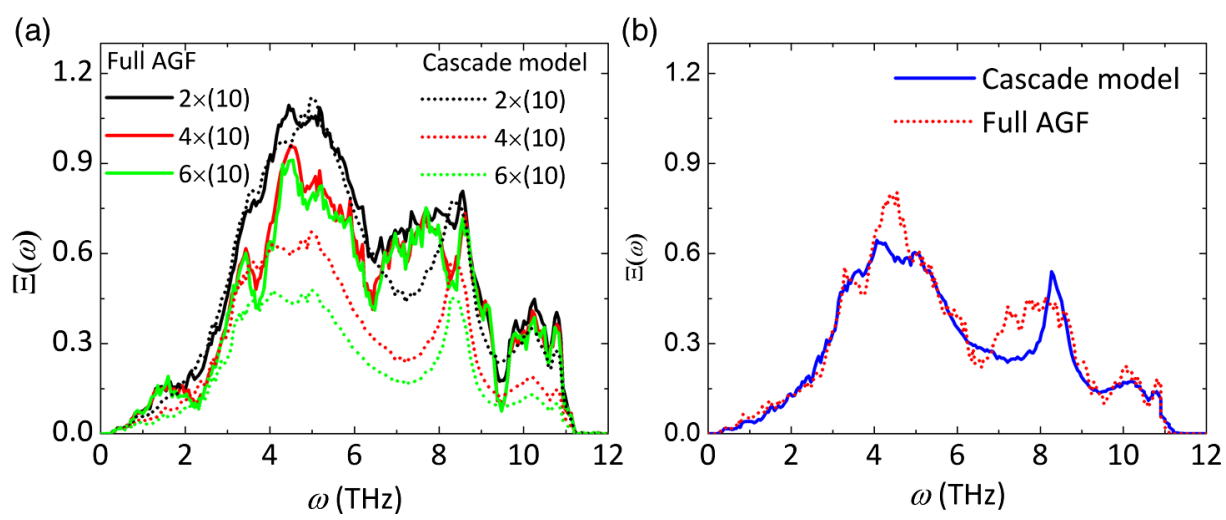

FIG. 6. Comparision between the phonon transmission obtained from the cascade model and full AGF calculation. (a) Periodic superlattices with different number of periods, where one period consists of a Si UL and a Ge UL denoted as "10." (b) The optimal aperiodic superlattice structure with total thickness of 10 UL. Here, the thickness of a unit layer is $5.43 \AA$.

terms of the descriptor). With increasing superlattice periods, the transmission of the full AGF calculation converges quickly, while the transmission of the cascade model keeps decreasing. This is understandable because the cascade model reflects only the incoherent phonon transport while the full AGF calculation captures the coherent phonon transport crossing multiple layers. The convergence of the full AGF transmission indicates that the effect of constructive interference in the periodic superlattices counteracts that of destructive interference. This suggests that there should be room to further reduce the phonon transmission and ITC if the balance between the constructive and destructive phonon interference can be altered. Note that these structures are the same as the ones calculated in Fig. 5(c), and thus explains the reason for the convergence.

Taking the optimal 10-UL superlattice for phonon transport from $\mathrm{Si}$ to $\mathrm{Si}$ with a fixed and equal $\mathrm{Si} / \mathrm{Ge}$ fraction as an example, (1101010001) can be divided into four individual components including one (0110) and three (010). Figure 6(b) shows the comparison between the effective phonon transmission from the cascade model and the transmission obtained from full AGF calculation. We find that the cascade model reproduces the general trend and magnitude of the transmission functions although there are certainly some differences in the details. The agreement suggests that the constructive phonon interference is suppressed relative to destructive interference by the aperiodic structure, and the phonon transmission approaches the incoherent phonon-transport limit, leading to the minimum ITC.

\section{CONCLUSIONS}

In conclusion, we identify the $\mathrm{Si} / \mathrm{Ge}$ composite interfacial structures that minimize or maximize the ITC across $\mathrm{Si}-\mathrm{Si}$ and $\mathrm{Si}-\mathrm{Ge}$ interfaces by the developed framework combining the atomistic Green function and Bayesian optimization methods. The optimal structures are obtained by calculating only a few percent of the total candidate structures, considerably saving computational resources. The validity and capability of the method are demonstrated by identifying the thin interfacial structures with the optimal $\mathrm{Si} / \mathrm{Ge}$ configurations among all the possible candidates. Here, a hierarchical approach is adopted to keep the number of candidates within an affordable range, where we first perform optimization of a smaller system with full degrees of freedom, and then extend it to a larger system without increasing the number of candidates by reducing the degrees of freedom using the result of the smaller system. Note that the affordable number of candidates would increase with advances in computational resources and optimization algorithms.

Based on the finding that the interfacial structures with minimum ITC take the form of an aperiodic superlattice, we extend the search to thicker structures (up to $8.69 \mathrm{~nm}$ ) and identify nonintuitive structures whose ITCs are significantly smaller than those of the optimal periodic superlattices. The small ITC in the aperiodic structures originates from their degree of freedom to mutually adoptively balance the two competing effects, FabryPérot wave interference and interfacial particle scattering, which reduces ITC as the thickness of the constituent layers in the superlattice increases and decreases, respectively. As a result, the optimal aperiodic structure is found to restrain the constructive phonon interference, delaying the convergence of the coherent heat conduction reduction. Here, we choose a Si-Ge system because it is the most studied superlattice structure in terms of thermal transport $[35,49,55,56]$. The developed method is applicable to any system, and systems with better control over the composition, such as III-V systems [16,57] and oxides $[19,48]$, may be the first to benefit from the method in practice. The present work shows the effectiveness and advantage of material informatics in designing nanostructures to control heat conduction. 


\section{ACKNOWLEDGMENTS}

This work was supported in part by the "Materials research by Information Integration" Initiative $\left(\mathrm{MI}^{2} \mathrm{I}\right)$ project and CREST Grant No. JPMJCR16Q5 from Japan Science and Technology Agency (JST), and KAKENHI (Grants No. 16H04274 and No. 15K17982). This research used computational resources provided by Information Initiative Center at Hokkaido University and Academic Center for Computing and Media Studies at Kyoto University through the HPCI System Research Project (Project No. hp160161), and those provided by the Institute for Solid State Physics, the University of Tokyo.

[1] R. Prasher, Thermal Interface Materials: Historical Perspective, Status, and Future Directions, Proc. IEEE 94, 1571 (2006).

[2] A. Faghri, Review and Advances in Heat Pipe Science and Technology, J. Heat Transfer 134, 123001 (2012).

[3] H. N. Chaudhry, B. R. Hughes, and S. A. Ghani, A Review of Heat Pipe Systems for Heat Recovery and Renewable Energy Applications, Renew. Sustain. Energ. Rev. 16, 2249 (2012).

[4] Z. T. Tian, S. Lee, and G. Chen, Heat Transfer in Thermoelectric Materials and Devices, J. Heat Transfer 135, 061605 (2013).

[5] O. Kaynakli, A Review of the Economical and Optimum Thermal Insulation Thickness for Building Applications, Renew. Sustain. Energ. Rev. 16, 415 (2012).

[6] S. Volz, J. Shiomi, M. Nomura, and K. Miyazaki, Heat Conduction in Nanostructured Materials, J. Therm. Sci. Technol. 11, JTST0001 (2016).

[7] P. M. Norris, N. Q. Le, and C. H. Baker, Tuning Phonon Transport: From Interfaces to Nanostructures, J. Heat Transfer 135, 061604 (2013).

[8] Z. Tian, K. Esfarjani, and G. Chen, Enhancing Phonon Transmission Across a $\mathrm{Si} / \mathrm{Ge}$ Interface by Atomic Roughness: First-Principles Study with the Green's Function Method, Phys. Rev. B 86, 235304 (2012).

[9] S. Merabia and K. Termentzidis, Thermal Boundary Conductance Across Rough Interfaces Probed by Molecular Dynamics, Phys. Rev. B 89, 054309 (2014).

[10] Y. Liu, C. Hu, J. Huang, B. G. Sumpter, and R. Qiao, Tuning Interfacial Thermal Conductance of Graphene Embedded in Soft Materials by Vacancy Defects, J. Chem. Phys. 142, 244703 (2015).

[11] P. K. Schelling, S. R. Phillpot, and P. Keblinski, Kapitza Conductance and Phonon Scattering at Grain Boundaries by Simulation, J. Appl. Phys. 95, 6082 (2004).

[12] S.-H. Ju and X.-G. Liang, Investigation on Interfacial Thermal Resistance and Phonon Scattering at Twist Boundary of Silicon, J. Appl. Phys. 113, 053513 (2013).

[13] M. Sakata, T. Hori, T. Oyake, J. Maire, M. Nomura, and J. Shiomi, Tuning Thermal Conductance Across Sintered Silicon Interface by Local Nanostructures, Nano Energy 13, 601 (2015).

[14] M. Sakata, T. Oyake, J. Maire, M. Nomura, E. Higurashi, and J. Shiomi, Thermal Conductance of Silicon Interfaces
Directly Bonded by Room-Temperature Surface Activation, Appl. Phys. Lett. 106, 081603 (2015).

[15] K. Zheng, F. Sun, X. Tian, J. Zhu, Y. Ma, D. Tang, and F. Wang, Tuning the Interfacial Thermal Conductance between Polystyrene and Sapphire by Controlling the Interfacial Adhesion, ACS Appl. Mater. Interfaces 7, 23644 (2015).

[16] M. N. Luckyanova, J. Garg, K. Esfarjani, A. Jandl, M. T. Bulsara, A. J. Schmidt, A. J. Minnich, S. Chen, M. S. Dresselhaus, Z. Ren, E. A. Fitzgerald, and G. Chen, Coherent Phonon Heat Conduction in Superlattices, Science 338, 936 (2012).

[17] J. Garg and G. Chen, Minimum Thermal Conductivity in Superlattices: A First-Principles Formalism, Phys. Rev. B 87, 140302(R) (2013).

[18] Y. Chen, D. Li, J. R. Lukes, Z. Ni, and M. Chen, Minimum Superlattice Thermal Conductivity from Molecular Dynamics, Phys. Rev. B 72, 174302 (2005).

[19] J. Ravichandran, A. K. Yadav, R. Cheaito, P. B. Rossen, A. Soukiassian, S. J. Suresha, J. C. Duda, B. M. Foley, C. H. Lee, Y. Zhu, A. W. Lichtenberger, J. E. Moore, D. A. Muller, D. G. Schlom, P. E. Hopkins, A. Majumdar, R. Ramesh, and M. A. Zurbuchen, Crossover from Incoherent to Coherent Phonon Scattering in Epitaxial Oxide Superlattices, Nat. Mater. 13, 168 (2014).

[20] P. Němec and P. Malý, Temperature Dependence of Coherent Phonon Dephasing in $\mathrm{CsPbCl}_{3}$ Nanocrystals, Phys. Rev. B 72, 235324 (2005).

[21] X. Li and R. Yang, Equilibrium Molecular Dynamics Simulations for the Thermal Conductivity of $\mathrm{Si} / \mathrm{Ge}$ Nanocomposites, J. Appl. Phys. 113, 104306 (2013).

[22] S. Ju and X. Liang, Detecting the Phonon Interference Effect in $\mathrm{Si} / \mathrm{Ge}$ Nanocomposite by Wave Packets, Appl. Phys. Lett. 106, 203107 (2015).

[23] K. Rajan, Materials Informatics: The Materials "Gene" and Big Data, Annu. Rev. Mater. Res. 45, 153 (2015).

[24] K. Rajan, Materials Informatics, Mater. Today 15, 470 (2012).

[25] A. Agrawal and A. Choudhary, Perspective: Materials Informatics and Big Data: Realization of the "Fourth Paradigm" of Science in Materials Science, APL Mater. 4, 053208 (2016).

[26] D. S. Wishart, Bioinformatics in Drug Development and Assessment, Drug metabolism reviews 37, 279 (2005).

[27] T. L. Blundell, B. L. Sibanda, R. W. Montalvao, S. Brewerton, V. Chelliah, C. L. Worth, N. J. Harmer, O. Davies, and D. Burke, Structural Biology and Bioinformatics in Drug Design: Opportunities and Challenges for Target Identification and Lead Discovery, Phil. Trans. R. Soc. B 361, 413 (2006).

[28] N. Adams and P. Murray-Rust, Engineering Polymer Informatics: Towards the Computer-Aided Design of Polymers, Macromol. Rapid Commun. 29, 615 (2008).

[29] S. Kiyohara, H. Oda, K. Tsuda, and T. Mizoguchi, Acceleration of Stable Interface Structure Searching Using a Kriging Approach, Jpn. J. Appl. Phys. 55, 045502 (2016).

[30] P. B. Wigley, P. J. Everitt, A. van den Hengel, J. W. Bastian, M. A. Sooriyabandara, G. D. McDonald, K. S. Hardman, 
C. D. Quinlivan, P. Manju, C. C. Kuhn, I. R. Petersen, A. N. Luiten, J. J. Hope, N. P. Robins, and M. R. Hush, Fast Machine-Learning Online Optimization of UltraCold-Atom Experiments, Sci. Rep. 6, 25890 (2016).

[31] A. Seko, A. Togo, H. Hayashi, K. Tsuda, L. Chaput, and I. Tanaka, Prediction of Low-Thermal-Conductivity Compounds with First-Principles Anharmonic Lattice-Dynamics Calculations and Bayesian Optimization, Phys. Rev. Lett. 115, 205901 (2015).

[32] J. Carrete, W. Li, N. Mingo, S. Wang, and S. Curtarolo, Finding Unprecedentedly Low-Thermal-Conductivity HalfHeusler Semiconductors via High-Throughput Materials Modeling, Phys. Rev. X 4, 011019 (2014).

[33] H. Zhang and A. J. Minnich, The Best Nanoparticle Size Distribution for Minimum Thermal Conductivity, Sci. Rep. 5, 8995 (2015).

[34] X. Li and R. Yang, Size-Dependent Phonon Transmission Across Dissimilar Material Interfaces, J. Phys. Condens. Matter 24, 155302 (2012).

[35] W. Zhang, T. S. Fisher, and N. Mingo, Simulation of Interfacial Phonon Transport in Si-Ge Heterostructures Using an Atomistic Green's Function Method, J. Heat Transfer 129, 483 (2007).

[36] J.S. Wang, J. Wang, and J. T. Lü, Quantum Thermal Transport in Nanostructures, Eur. Phys. J. B 62, 381 (2008).

[37] T. Ueno, T. D. Rhone, Z. Hou, T. Mizoguchi, and K. Tsuda, сомво: An Efficient Bayesian Optimization Library for Materials Science, Mater. Discovery 4, 18 (2016).

[38] R. Landauer, Electrical Resistance of Disordered OneDimensional Lattices, Philos. Mag. 21, 863 (1970).

[39] I. M. Khalatnikdrov and I. N. Adamenko, Theory of the Kapitza Temperature Discontinuity at a Solid Body-Liquid Helium Boundary, J. Exp. Theor. Phys. 36, 391 (1973).

[40] J. Tersoff, Modeling Solid-State Chemistry: Interatomic Potentials for Multicomponent Systems, Phys. Rev. B 39, 5566 (1989).

[41] J. Tersoff, Erratum: Modeling Solid-State Chemistry: Interatomic Potentials for Multicomponent Systems, Phys. Rev. B 41, 3248(E) (1990).

[42] See Supplemental Material at http://link.aps.org/ supplemental/10.1103/PhysRevX.7.021024 for detailed discussion about (A) Dependence of phonon transmission and conductance on the $k$-mesh size in atomistic Green's function calculation. (B) Dependence of the optimization performance on the number of candidates added at each round. (C) Fabry-Pérot oscillations of phonon transmission in one dimensional chain. (D) Generation of structure candidates for large cross section area system.
[43] D. R. Jones, A Taxonomy of Global Optimization Methods Based on Response Surfaces, J. Global Optim. 21, 345 (2001).

[44] A. Rahimi and B. Recht, Random Features for Large-Scale Kernel Machines, in Proceedings of the 20th International Conference on Neural Information Processing Systems (Curran Associates Inc., Vancouver, British Columbia, 2007), pp. 1177-1184.

[45] Z. Yang, A. J. Smola, L. Song, and A. G. Wilson, A la Carte-Learning Fast Kernels, in Proceedings of the Eighteenth International Conference on Artificial Intelligence and Statistics (Journal of Machine Learning Research: Workshop and Conference Proceedings, San Diego, CA, 2015), pp. 1098-1106.

[46] D. P. Kingma and J. L. Ba, Adam: A Method for Stochastic Optimization, arXiv:1412.6980.

[47] B. Qiu, G. Chen, and Z. Tian, Effects of Aperiodicity and Roughness on Coherent Heat Conduction in Superlattices, Nanoscale Micro. Thermophys. Eng. 19, 272 (2015).

[48] H. N. Lee, H. M. Christen, M. F. Chisholm, C. M. Rouleau, and D. H. Lowndes, Strong Polarization Enhancement in Asymmetric Three-Component Ferroelectric Superlattices, Nature (London) 433, 395 (2005).

[49] H. Zhao and J. B. Freund, Lattice-Dynamical Calculation of Phonon Scattering at Ideal Si-Ge Interfaces, J. Appl. Phys. 97, 024903 (2005).

[50] P. Hyldgaard, Resonant Thermal Transport in Semiconductor Barrier Structures, Phys. Rev. B 69, 193305 (2004).

[51] P. E. Hopkins, P. M. Norris, M. S. Tsegaye, and A. W. Ghosh, Extracting Phonon Thermal Conductance Across Atomic Junctions: Nonequilibrium Green's Function Approach Compared to Semiclassical Methods, J. Appl. Phys. 106, 063503 (2009).

[52] J. Wang, L. Li, and J.-S. Wang, Tuning Thermal Transport in Nanotubes with Topological Defects, Appl. Phys. Lett. 99, 091905 (2011).

[53] S. Datta, Quantum Transport: Atom to Transistor (Cambridge University Press, Cambridge, England, 2005).

[54] S. Datta, Electronic Transport in Mesoscopic Systems (Cambridge University Press, Cambridge, England, 1995).

[55] Z. T. Tian, K. Esfarjani, and G. Chen, Green's Function Studies of Phonon Transport Across Si/Ge Superlattices, Phys. Rev. B 89, 235307 (2014).

[56] K.-H. Lin and A. Strachan, Thermal Transport in SiGe Superlattice Thin Films and Nanowires: Effects of Specimen and Periodic Lengths, Phys. Rev. B 87, 115302 (2013).

[57] W. Braun, A. Trampert, L. Daweritz, and K. H. Ploog, Nonuniform Segregation of Ga at AlAs/GaAs Heterointerfaces, Phys. Rev. B 55, 1689 (1997). 